\title{
MICROSSERVIÇOS SEGUROS - UMA REVISÃO APLICANDO A TEORIA DO ENFOQUE METAANALITICO CONSOLIDADO
}

\author{
Phillipy Ferreira ${ }^{1}$, Welyngton Dal Prá ${ }^{1}$, Daniel Araújo ${ }^{1}$, \\ Gabriel de O. Alves ${ }^{1}$, Lucas M. C. e Martins ${ }^{1}$ e William F. Giozza ${ }^{1,2}$ \\ ${ }^{1}$ Laboratório de Tecnologias da Tomada de Decisão (LATITUDE.UnB) - Universidade de Brasília, Brasil \\ ${ }^{2}$ Departamento de Engenharia Elétrica (ENE) - Universidade de Brasília, Brasil
}

\begin{abstract}
RESUMO
A proposta deste artigo é abordar a relevância na aplicação da arquitetura de sistemas baseada em microsserviços com ênfase na questão dos desafios de implementar a segurança neste modelo. Partindo do impacto que isso trouxe a literatura acadêmica, foi realizada uma profunda análise bibliográfica utilizando a Teoria do Enfoque Meta-Analítico Consolidado para traçar inter-relações entre os estudos já realizados sobre segurança em microsserviços com o objetivo de identificar tendências, principais autores, centros de pesquisa, trabalhos relevantes e palavras-chave. A partir de uma base de 733 artigos do Scopus, alguns dos principais fatos encontrados por meio da análise de metadados bibliográficos foram que os autores de maior influência são Fowler, M., Newman, S. Buyya, R., Chen Y., e Zhang, Y, os países que mais publicaram sobre o tema foram China, USA e Alemanha e que o tema se apresenta ainda em ascensão na literatura acadêmica.
\end{abstract}

\section{PALAVRAS-CHAVE}

Microsserviços, Segurança, Revisão Bibliográfica, Bibliometria

\section{INTRODUÇÃO}

Há uma notável ascensão quanto ao estudo e emprego de microsserviços na arquitetura de sistemas (Kratzke, 2018). Empresas como Amazon, Netflix (Newman, 2015), Google, Twitter e Uber com grande impacto e influência na área de tecnologia passaram a adotar esse tipo de solução (Kratzke, 2018), despertando ainda mais atenção quanto ao tema.

Considerando a grande relevância e predominância do modelo arquitetural de sistemas baseados em microsserviços (Kratzke, 2018), alguns desafios como a questão da segurança se impõem (Alshuqayran et al., 2016) diante da transformação da arquitetura tradicional de sistemas complexos monolíticos para diversas microestruturas simples cuja complexidade é transferida agora para a interligação e controle destes microssistemas (Yarygina e Bagge, 2018).

Alguns desafios de pesquisa presentes na literatura são: a comunicação/integração entre os serviços, performance, tolerância a falhas, segurança, log e monitoração e operações de implantação (Alshuqayran et al., 2016).

Após a realização de levantamento bibliográfico em bases científicas como Scopus, Web of Science (WoS) e Google Scholar foi constatado que há uma ampla bibliografia a respeito do tema, por conseguinte apresenta-se a necessidade de uma revisão profunda sobre o aspecto da segurança, dado que é um dos assuntos centrais na literatura (Alshuqayran et al., 2016) (Chondamrongkul et al., 2020).

O objetivo deste estudo é realizar uma revisão da literatura a fim de consolidar e validar interrelações verificadas numa investigação em profundidade fundamentada em leis bibliométricas e suas análises, com a finalidade de evidenciar fatores, relações e tendências acerca da segurança em microsserviços. As seguintes questões de pesquisa guiaram este estudo: QP. 1) Quais os principais autores relacionados a segurança em microsserviços? QP. 2) Quais os países mais prolíficos em segurança em microsserviços na literatura? QP. 3) Quais os principais centros de pesquisa e onde estão localizados? QP. 4) Quais as palavras-chave mais recorrentes sobre segurança em microsserviços? 
A contribuição deste trabalho é identificar tendências e informações bibliométricas relevantes baseadas em uma profunda análise bibliográfica por meio da Teoria do Enfoque Meta-analítico Consolidado (TEMAC) (Mariano e Rocha, 2017).

Algumas das principais descobertas foram que os autores de maior influência são Fowler, M., Newman, S. Buyya, R., Chen Y., e Zhang, Y, os países que mais publicaram sobre o tema foram China, USA e Alemanha e que há grande oportunidade de pesquisa sobre o assunto.

Na próxima seção é apresentada uma introdução ao tema de segurança de microsserviços, com histórico, desafios e abordagem que será utilizada no trabalho. Na Seção 3 é discutida a metodologia TEMAC que foi utilizada na sistematização. A Seção 4 mostra a aplicação da metodologia, subdivida em 4.1 Preparação da Pesquisa, 4.2 Inter-relação e análise de dados e 4.3 Validação da Pesquisa. Concluindo o artigo na Seção 5.

\section{CONTEXTO}

Microsserviços, segundo Newman (2015), são pequenos serviços autônomos trabalhando em conjunto. O autor também lista alguns princípios desse modelo, tais como: modelagem em torno de conceitos ou domínios de negócio, cultura de automação, abstração de detalhes internos de implementação, descentralização e autonomia na tomada de decisão, implantação independente, isolamento de falhas e alta capacidade de monitoração.

Algumas das principais vantagens desse modelo são a escalabilidade, a implantação independente, a facilidade de manutenção, além de ampliar as vantagens já obtidas com a adoção dos modelos de Arquitetura Orientada a Serviços (SOA) e de Sistemas Distribuídos (Newman, 2015) (Kratzke, 2018).

Um dos temas de maior relevância na bibliografia de microsserviços é a questão da segurança (Alshuqayran et al., 2016) (Chondamrongkul et al., 2020), visto que: 1) a granularidade da arquitetura de microsserviços é composta de serviços executados na rede; 2) os serviços são geralmente executados em contêineres baseados em nuvem, portanto a segurança do sistema é fortemente baseada na confiança nesses contêineres; 3) os microsserviços devem confiar que o controle de autenticação e autorização farão seu trabalho adequadamente; 4) como os serviços estão dispersos na rede, a transferência de dados sobre a rede fica vulnerável a ataques (Chondamrongkul et al., 2020).

A partir da comparação de alguns modelos de revisão bibliográfica como a Revisão Qualitativa, Revisão Integrativa, Revisão Sistemática e Meta-análises, verificou-se que as meta-análises e revisões sistemáticas estão mais limitadas a levantamentos quantitativos fechados em análises estatísticas, possibilitando reduzir o viés de outros estudos ou ainda integrar vários estudos primários, enquanto as demais revisões, ainda que permitam realizar também análises qualitativas, permanecem no escopo de sumarização ou de revisão de teorias, estudos e métodos.

Sendo assim definiu-se por utilizar a Teoria do Enfoque Meta-Analítico Consolidado (TEMAC), visto que este método permite adotar abordagens qualitativas e quantitativas (Mariano e Rocha, 2017) utilizando passos definidos e padronizados para a revisão e análise de metadados bibliográficos, possibilitando a identificação de tendências, áreas de enfoque, cooperação e trabalhos relevantes para a área na literatura acadêmica.

\section{METODOLOGIA}

O enfoque meta analítico proposto pelo TEMAC pretende traçar correlações e tendências em artigos por meio de um método desenhado em etapas que pode ser auxiliado por ferramentas como LibbreOffice Calc, VOSviewer, TagCrowd e Publish or Perish (Perdigão et al., 2019).

A teoria do enfoque meta-analítico consolidado (Mariano e Rocha, 2017) apresenta 3 passos que devem ser seguidos a fim de obter resultados analíticos: Etapa 1 - A preparação da pesquisa, delimitação da abrangência e seleção da base de dados; Etapa 2 - A apresentação da interrelação dos dados e a Etapa 3 - Detalhamento, modelo integrador e validação por evidências.

O TEMAC (Mariano e Rocha, 2017) também permite aplicar conceitos de bibliometria, campo da Ciência da Informação (Beuren e Souza, 2008) que baseia-se em um conjunto de regras e princípios aplicando métodos matemáticos e estatísticos. Algumas das das principais leis e princípios bibliométricos utilizadas foram: a Lei do Elitismo que determinada por $\sqrt{k}$ onde $\mathrm{k}$ é a quantidade total de autores na área, sendo que esse cálculo 
determinaria a elite de autores na área, também a Lei do 80/20 (Princípio de Pareto) para estimar o grau de relevância de palavras-chave, autores, acoplamento bibliográfico, análise de co-citações em dada área do conhecimento (Guedes e Borschiver, 2005).

\section{EXECUTANDO O TEMAC}

Conforme a metodologia TEMAC (Mariano e Rocha, 2017) aplicada na realização desta análise, serão executados os 3 passos do método: 1) preparação, 2) análise e 3) validação. Descritos a seguir:

\subsection{Preparação da Pesquisa}

Uma das questões levantadas pelo TEMAC (Mariano e Rocha, 2017) é: Qual o descritor, string ou palavra-chave da pesquisa? Para encontrar o melhor descritor foi utilizada a seguinte estratégia por meio de um algoritmo: 1. Pesquisa dos principais termos utilizados na literatura nas bases Scopus, WoS e Google Scholar; 2. Validação nos artigos mais relevantes dos principais termos utilizados; 3. Pesquisa com os termos levantados e avaliação dos resultados; 4. Incorporação dos termos "AND", "OR" e "NOT" para limitar os resultados encontrados; 5. Validação dos resultados encontrados: se correspondiam ao foco desta revisão; 6 . Após refinamento, executar o passo 3 novamente até que a condição do passo 5 seja constatada.

Após a primeira avaliação, a pesquisa foi realizada apenas com os termos "MICROSERVICE" e "SECURITY". Dentre os artigos retornados foi encontrado o estudo sistemático de Alshuqayran (2016) que lista um conjunto de termos relacionados a segurança em microsserviços como: "secure", "authentication”, "authorization", "OAuth", "OAuth2", "encryption", "vulnerability" e "attack". Tais termos foram integrados como sinônimos de segurança para nosso descritor de busca.

Dentre os resultados apresentados foi verificado que havia muitos trabalhos relacionados a Internet of Things(IoT), entretanto foi delimitado no escopo da revisão que a arquitetura de microsserviços que deseja-se abordar é a de sistemas de estrutura convencional (não-IoT) (Washizaki et al., 2020), excluindo assim microsserviços aplicados a Internet das Coisas, devido às particularidades desse modelo, ainda que se tenha observado uma abundante bibliografia.

Após algumas repetições dos passos descritos, foi encontrada uma string satisfatória para o escopo do trabalho, e dentro do resultado abrangente encontrado, foi verificada a presença de vários artigos com relevância acadêmica e coerentes com o objetivo desta revisão. O descritor de busca utilizado nas bases de dados científicos foi:

$$
\begin{gathered}
\text { ( "MICROSERVICE" OR "MICROSERVICES" ) AND ( "SECURITY" OR "SECURE" OR } \\
\text { "AUTHENTICATION" OR "AUTHORIZATION" OR "OAUTH" OR "OAUTH2" OR "ENCRYPTION" OR } \\
\text { "VULNERABILITY" OR "ATTACK" ) AND NOT ( "IOT" OR "INTERNET OF THINGS" ) }
\end{gathered}
$$

Seguindo a primeira etapa do TEMAC (Mariano e Rocha, 2017) é importante delimitar o espaço-tempo da pesquisa, assim o primeiro artigo encontrado com o termo microservice é de 2012, "A semantic metadirectory of services based on web mining techniques" de Fernández-Villamor \& et al, com apenas 3 citações encontradas, o tema continuou com cerca de 2 publicações por ano, sem nenhuma publicação de impacto, até 2015, quando o livro "Building Microservices: Designing Fine-Grained Systems" de Sam Newman é publicado. O termo microservice ganha relevância após esta data, comprovando o grande impacto da obra. Logo percebe-se que os trabalhos de maior impacto acadêmico passam a ser publicados após 2015, conforme será apresentado posteriormente, desta forma a pesquisa foi refinada para trazer resultados entre os anos de 2015 até 2020.

Observando a Figura 1 é possível perceber que o tema encontra-se em ascensão, apresentando uma curva ascendente de 2015 a 2019, sendo que o ano de 2020 já possui cerca de $60 \%$ da quantidade de total de artigos publicados em 2019 em menos de 8 meses, o que demonstra que esta revisão ainda é relevante para a área. 


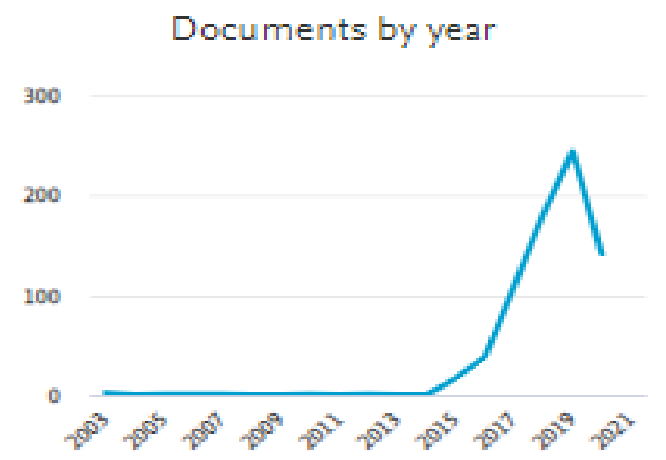

Figura 1. Gráfico de número de publicações por ano Fonte: Própria - Dados: Extraído da plataforma Scopus

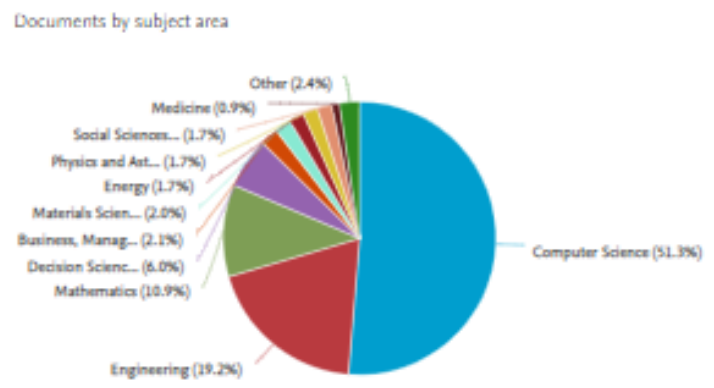

Figura 2. Gráfico de áreas mais relevantes na busca Fonte: Própria - Extraído da plataforma Scopus

Após análise das principais bases de dados científicas, tais como Web of Science, Scopus, Microsoft Academic e Google Scholar visto que as bases têm conteúdos e algoritmos diferentes (Rovira et al., 2019), optou-se por utilizar somente a plataforma Scopus, principalmente pelo fato de possuir melhor cobertura de revistas de ciência e tecnologia, abranger mais conteúdo global, cerca de $60 \%$ do conteúdo é de fora dos EUA, e possuir melhor cobertura temporal dos últimos 5 anos (Mariano \& Rocha, 2017).

Após análise realizada no Scopus, as áreas com maior destaque quanto ao assunto são: Computer Science, Engineering, Mathematics e Decision Sciences conforme a Figura 2. Esse filtro possibilitou restringir os resultados, pois haviam sido identificados trabalhos que envolviam o tema de segurança como principal assunto, como por exemplo serviços de saúde, e utilizavam microservice dentro de seu artigos, o que poluiu a base de pesquisa.

Segundo Zupic e Cater (2014) uma das tarefas essenciais para o avanço numa determinada linha de pesquisa é sintetizar os achados de pesquisas passadas. Utilizando técnicas de bibliometria visando investigar e analisar a literatura científica e suas relações, tais relações serão descritas a seguir.

\subsection{Apresentação e Inter-Relação dos Dados}

Partindo da própria ferramenta de análise de resultados do Scopus conforme a Figura 3, é possível identificar que EUA, Alemanha e China são os países que mais publicam sobre o tema, vale ressaltar que apesar de densidade demográfica mais representativa na China e EUA, e mesmo assim a Alemanha figura em segundo lugar, entretanto essa análise não é foco deste trabalho. Neste gráfico (Documents by Country) também vale observar que o Brasil é o único país do Hemisfério Sul entre os 10 que mais publicam. Analisando o segundo gráfico (Documents by sponsor) pode-se verificar que os 3 principais apoiadores do assunto são instituições dos EUA, China e Europa, na proporção direta com a quantidade de trabalhos produzidos em cada uma dessas regiões. Quanto às instituições associadas o que se destaca é que as 4 principais instituições são alemãs (Documents by affiliation), com destaque para a Universidade de Potsdam, que possui como afiliados os autores que mais publicaram (Documents by author) nos últimos 5 anos: Meinel, C., Sukmana, M. I. H., Torkura K.A. e Cheng, F. que possuem diversos trabalhos em conjunto sobre cloud, o que evidencia ser um importante centro de pesquisa sobre temas relacionados à segurança e microsserviços: 

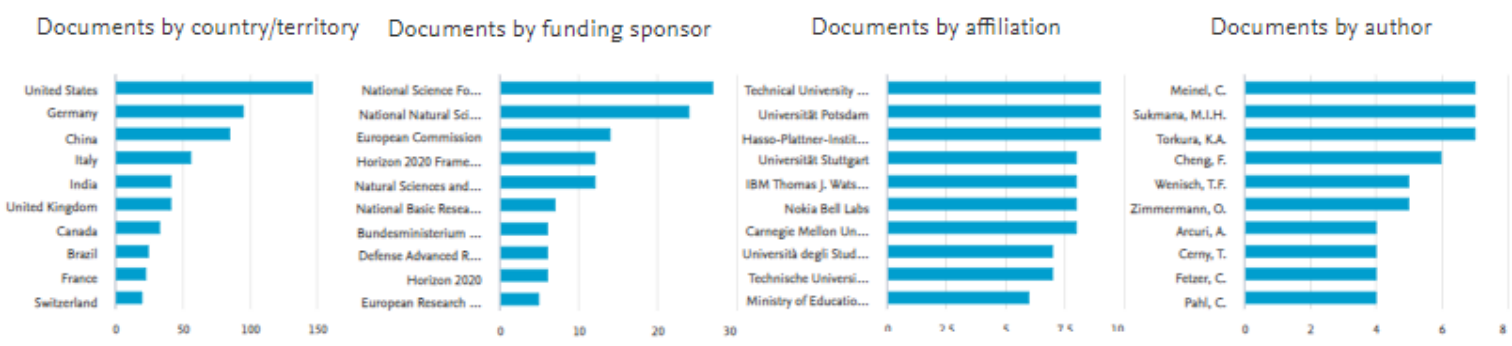

Figura 3. Análise de resultados de busca na Plataforma Scopus

Fonte: Própria - Extraído da plataforma Scopus

A Figura 3 apresenta o diagrama de rede de co-citação, segundo Zupic e Cater (2014) a probabilidade de similaridade de conteúdo é maior, quanto mais itens são citados em conjunto, portanto a análise deste diagrama irá apresentar os autores que possuem linha de pesquisa similar.

Observa-se a presença de 5 grupos de autores que são comumentes co-citados em trabalhos acadêmicos, é possível validar que há bastante dispersão dos dados, sendo os autores mais citados, nesta ordem, Fowler, M., Newman, S. Buyya, R., Chen Y., e Zhang, Y. dando ênfase para a difusão deste tema por autores da Oceania, Ásia, América e Europa, conforme também pode-se observar na Figura 4.

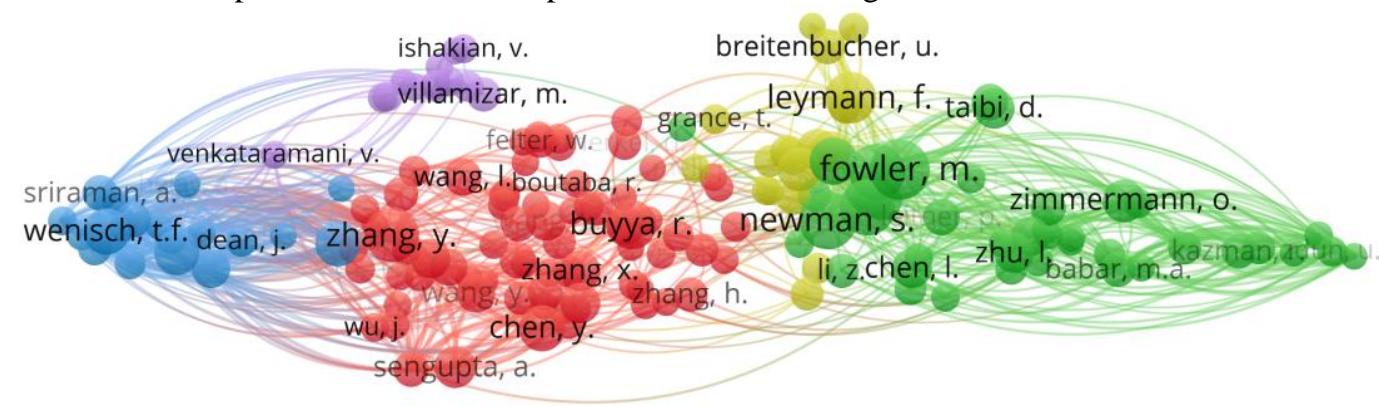

Figura 4. Diagrama de rede do software VOSviewer de co-citação de autores

Fonte: Própria - Extraído da plataforma Scopus

Conforme descrito na seção 3.1, Newman foi um dos responsáveis por popularizar o termo microservice, portanto muitos trabalhos partem da definição dada por ele e complementada por Fowler, mostrando alto grau de citação dos livros destes autores em todos os grupos apresentados. Entretanto a divisão em outros 4 grupos comprova que o assunto está sendo abordado de diferentes formas, que a cada momento a distância dos autores da fonte inicial está maior, gerando novos grupos com outras referências e possíveis novas linhas de pesquisa. Um exemplo claro é Buyya, R., o autor mais citado do grupo central, juntamente com Chen, Y. e Zhang, Y.. Também observam-se outros 3 grupos com grande relevância para o tema, dos autores Wenisch, T. e Sriraaman, A. no grupo a esquerda, Ishakian, V. e Villamizar, M. no grupo superior esquerdo e Leymann, F. e Breitenbucher, U. no grupo superior direito representam possíveis novas abordagens para o tema.

Além destas divisões claras é possível notar que o tema continua em expansão, endossando a análise realizada na seção 3.1 de que o número de publicações a respeito do tema está em ascensão. O distanciamento dos autores como Zimmermann, O., Zhu, L. e Kazman, R., de seu atual núcleo, pode representar a criação de um novo grupo, o mesmo ocorre com Sengupta, A., Chen, Y. e Wu. J., provando o contínuo crescimento do tema e formas de abordá-lo.

Sobre o relacionamento de co-autoria entre os países, a análise dos dados gerou o mapa de calor apresentado na Figura 5 de acordo com a origem geográfica dos autores que possuem publicações em conjunto, esta análise não tem objetivo avaliar quais países possuem maior número de publicação, mas sim avaliar a relação de co-autoria entre as nações.

Podem ser observados 3 grupos principais de países com grandes quantidades de publicações e grande relação de co-autoria, sendo o maior deles liderado por Estados Unidos e China, além de Índia, Austrália, Canadá e Chile. Em seguida temos a Alemanha com grande quantidade de publicações, porém menor relação de co-autoria, sendo seu principal elo com o Reino Unido, que por sua vez integra o grupo mais central, liderado pela Itália e com importante presença também do Brasil e da França. A posição centralizada deste grupo indica 
que apesar de terem grande relação de co-autoria entre si, eles também possuem uma boa relação com os países dos grupos vizinhos, o que mostra a grande variedade de linhas de pesquisa produzida por autores desses países. Uma particularidade é a Coréia do Sul, que possui quantidade relevante de produção na área, porém está isolada, o que indica que não co-autoria com os outros grupos.

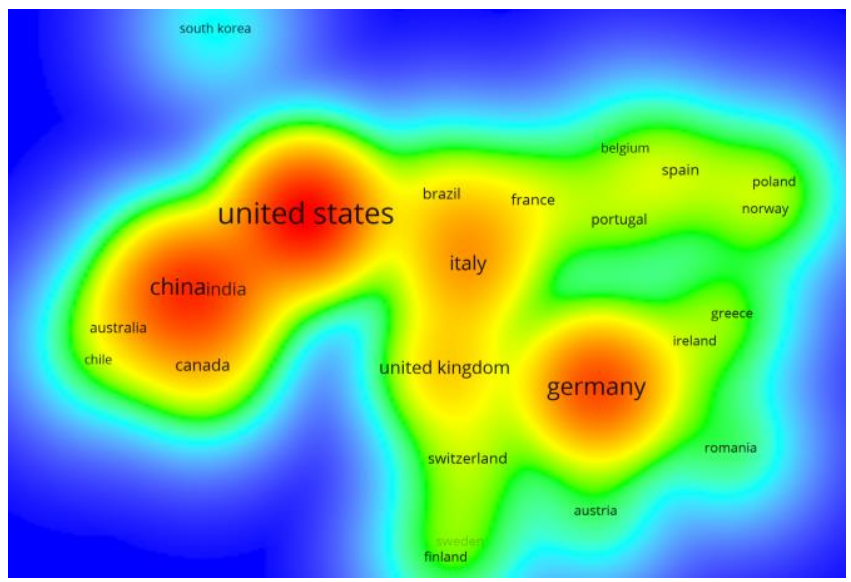

Figura 5. Diagrama de rede do software VOSviewer de co-autoria de países Fonte: Própria - Extraído da plataforma Scopus

A fim de entender quais são as frentes de pesquisa foi utilizada a Figura 6, que apresenta o mapa de calor de acoplamento bibliográfico. $\mathrm{O}$ acoplamento bibliográfico utiliza o número de referências compartilhadas entre dois documentos para identificar similaridade entre eles, quanto mais sobreposição bibliográfica mais similares os documentos tendem a ser (ZUPIC e CATER, 2014). Portanto, através desse mapa é possível destacar as principais frentes de pesquisa a respeito do tema.

Para fins desta análise, foram avaliados os últimos 3 anos de publicações, pois desta forma é possível revelar quais são as abordagens mais consolidadas, as que estão se fortalecendo e, ainda, identificar quais estão surgindo e não possuem citação, além de também excluir linhas de pesquisa que foram iniciadas e abandonadas pela comunidade.

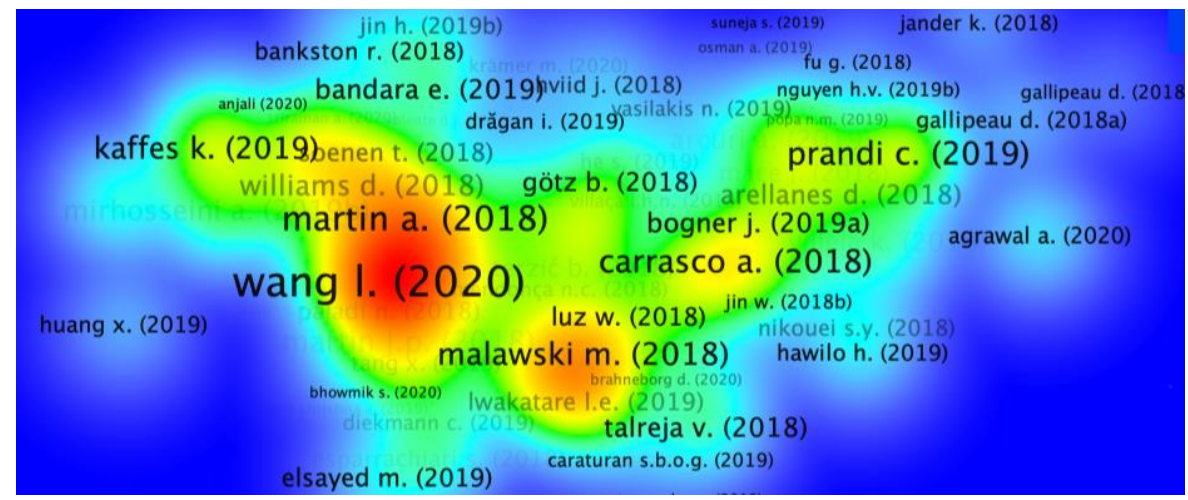

Figura 6. Mapa de calor do software VOSviewer de acoplamento bibliográfico de documentos Fonte: Própria - Extraído da plataforma Scopus

O mapa de calor da Figura 6 possui um total de 459 documentos, número menor que o apresentado na Figura 3 de co-citação, portanto não contempla todos os documentos já analisados. Esta análise consolida a aderência entre os trabalhos encontrados na base do SCOPUS, excluindo tipos de publicações que não são indexadas em bases científicas, tais como livros, artigos não científicos e outras. 
O primeiro destaque a se observar é Wang, I e colabores com o artigo "Peeking Behind the Curtains of Serverless Platforms", 16\% dos documentos possuem alto acoplamento com esse trabalho, em que discorre sobre o paradigma serverless computing, especificamente como APIs atualmente estão sendo construídas, explicando como as plataformas que oferecem esse serviço isolam as funcionalidades, sendo containers ou máquinas virtuais, enquanto são executadas e quais implicações de segurança devem ser levadas em consideração.

Num grupo próximo com outros 7\% dos documentos, é possível observar o trabalho de Martin, A e colaboradores de título "Docker ecosystem - Vulnerability Analysis", que possui certo grau de acoplamento com Wang, mas está mais interessado em apontar as vulnerabilidades em um ecossistema baseado em docker, apresentando casos reais de como essas vulnerabilidades podem ser exploradas e propondo possíveis soluções, além discutir a adoção de docker providos em PaaS.

Ambos os artigos envolvem microservice como a aplicação mais indicada para esses sistemas, destacando os ganhos de disponibilidade, escalabilidade horizontal e vertical e curtos ciclo de desenvolvimento, possibilitando a redução do custo para disponibilização de aplicações.

Em outro ponto da Figura 6 destaca-se Carrasco e colaboradores com o trabalho "Migrating towards Microservices: Migration and Architecture Smells", evidenciando o processo de migração para microservice para quem usa arquitetura tradicional (Yarygina e Bagge, 2018), o que demonstra que a arquitetura que utiliza microservice vem ganhando espaço(Kratzke, 2018). Esta frente de pesquisa corresponde a 9\% dos trabalhos, apresentando muitas comparações entre a arquitetura tradicional e os ganhos do uso do microservice e os desafios encontrados.

Com $11 \%$ dos trabalhos possuem como frente de pesquisa o estudo de casos sobre o uso de microservices, tais como implantações realizadas, resultados obtidos e desafios encontrados. Um trabalho a se destacar é o de Luz, W. e colaboradores, com o título "An experience report on the adoption of microservices in three Brazilian government institutions", em através de um estudo de casos evidencia os benefícios que a adoção de microservices trouxe diversos para as instituições.

Existem outras 9 frentes de pesquisa representadas pela Figura 6, entretanto cada uma destas representa cerca de $5 \%$ ou menos do total de documentos, portanto a grande distribuição de frentes de pesquisa indica que o tema ainda encontra-se em expansão e não possui alto referências consolidadas em todas as áreas de atuação, além de evidenciar que alguns grupos novos de pesquisa estão surgindo, apresentando acoplamento bibliográfico baixo com os atuais temas que atualmente estão em destaque.

\subsection{Detalhamento, Modelo Integrador e Validação por Evidências}

Um fator que evidencia a assertividade do modelo de dados escolhido para esta revisão meta-analítica é encontrar na bibliografia pelo menos 1 artigo de revisão sistemática (Mariano e Rocha, 2017). Na pesquisa realizada foram encontrados 14 artigos de revisão sistemática relacionados ao tema investigado Alshuqayran (2016) e Hassan (2019) por exemplo apresentam estudos sistemáticos sobre metadados e seus principais desafios, cujos apontamentos auxiliaram o direcionamento deste estudo.

Aplicando a Lei do Elitismo de Price (1963) verificamos que numa base de um total de 2322 autores na área, conforme a fórmula da Lei $\sqrt{2322}$ obtêm-se o resultado de 48 autores que seriam a elite da área e que, segundo esta lei, deveriam ser responsáveis por $50 \%$ das publicações na área. No entanto encontramos um total de apenas 174 artigos publicados por esses autores, ou seja, menos de $25 \%$, o que pode evidenciar que ainda não há uma elite formada na área, visto que a maioria dos autores é iniciante na área (Alvarado, 2009).

\section{CONCLUSÃO}

A partir dos estudos realizados seguindo a Teoria do Enfoque Meta-analítico Consolidado pode-se verificar que muito além da relevância dos desafios de segurança aplicada a arquitetura de microsserviços, este é um tema que ainda trás grandes oportunidades para pesquisa e aprofundamento considerando a complexidade envolvida em arquiteturas de microssistemas autônomos distribuídos em rede. 
A metodologia utilizada baseada em etapas também foi um grande facilitador na sistematização dos metadados encontrados nas bases científicas analisadas, com destaque para o Scopus, sendo assim, foi identificado que o TEMAC pode ser um grande aliado de pesquisadores (Cerqueira et al., 2020) que buscam analisar palavras-chave mais relevantes, principais autores e centros de pesquisa ou ainda aplicar técnicas bibliométricas a fim de identificar inter-relações e tendências relevantes.

O modelo de pesquisa utilizado sobre as bases de dados científicas também pode ser utilizado como base para outros estudos como ponto de partida ou direcionar ou também como modelo para revisar ou validar uma pesquisa.

Futuramente este estudo pode ser expandido utilizando mais bases de dados como o Microsoft Academy, ou ainda comparando dados do Scopus com os resultados do Google Scholar e o WOS e ainda incluir o subárea de Internet-of-Things que se mostrou com grande repercussão na literatura (Washizaki et al., 2020).

\section{REFERÊNCIAS}

Alshuqayran, N.; Ali, N.; Evans, R. ”A systematic mapping study in microservice architecture" Proceedings - IEEE 9th International Conference on Service-Oriented Computing and Applications, SOCA 2016

Alvarado, Rubén Urbizagástegui. (2009). Elitismo na literatura sobre a produtividade dos autores. Ciência da Informação, 38(2), 69-79. https://doi.org/10.1590/S0100-19652009000200006

Beuren, I. M.; Souza, J. C. d. "Em busca de um delineamento de proposta para classificação dos periódicos internacionais de contabilidade para o Qualis CAPES", Revista Contabilidade \& Finanças, 2008, vol.19, n.46, pp.44-58. https://doi.org/10.1590/S1519-70772008000100005.

Cerqueira, J. A. S. d. et al. "Exploratory Overview on Breaking CAPTCHAs Using the Theory of the Consolidated Meta-Analytic Approach," 15th Iberian Conference on Information Systems and Technologies (CISTI), Sevilla, Spain, 2020, pp. 1-6, doi: 10.23919/CISTI49556.2020.9140983.

Chondamrongkul, N.; Sun J.; Warren, I. "Automated Security Analysis for Microservice Architecture," IEEE International Conference on Software Architecture Companion (ICSA-C), Salvador, Brazil, 2020, pp. 79-82, doi: 10.1109/ICSAC50368.2020.00024.

Guedes, V. L. S.; Borschiver, S. "Bibliometria: uma ferramenta estatística para a gestão da informação e do conhecimento, em sistemas de informação, de comunicação e de avaliação científica e tecnológica", Encontro Nacional de Ciências da Informação, 6., Salvador/BA, 2005.

Hassan, S.; Bahsoon, R.; Kazman, R. "Microservice transition and its granularity problem: A systematic mapping study" School of Computer Science, University of Birminhgam, West Midlands, United Kingdom Software Engineering Institute (SEI)/CMU, Pittsburgh, PA, United States University of Hawaii, Honolulu, HI, United States, 2019.

Kratzke, N. "A Brief History of Cloud Application Architectures", Appl. Sci. 2018, 8, 1368. https://doi.org/10.3390/app8081368.

Mariano, A. S.; Rocha, M. "Revisão da literatura: Apresentação de uma abordagem integradora," XXVI Congreso Internacional de la Academia Europea de Dirección y Economía de la Empresa (AEDEM), Reggio Calabria, vol. 26, 2017.

Newman S. "Building Microservices", O’Reilly Media, 2015.

Perdigão, A.; Prado, F.; Mariano, A. S. "Uso de base de dados científica: um estudo exploratório por meio da Teoria do Enfoque Meta Analítico Consolidado -TEMAC", 2019.

Price, J. D. d. S. "Little science, big science", New York: Columbia University Press, 1963.

Rovira, C.; Codina, L.; Guerrero-Solé, F.; Lopezosa, C. "Ranking by Relevance and Citation Counts, a Comparative Study: Google Scholar, Microsoft Academic, WoS and Scopus", Future Internet, 2019. doi:10.3390/fi11090202.

Washizaki, H.; Ogata, S.; Hazeyama, A.; Okubo, T.; Fernandez, E. B. ; Yoshioka, N. "Landscape of Architecture and Design Patterns for IoT Systems," in IEEE Internet of Things Journal, doi: 10.1109/JIOT.2020.3003528.

Yarygina T.; Bagge, A. H. "Overcoming Security Challenges in Microservice Architectures," IEEE Symposium on Service-Oriented System Engineering (SOSE), Bamberg, 2018, pp. 11-20, doi: 10.1109/SOSE.2018.00011.

Zupic, I.; Cater, T. "Bibliometric methods in management and organization", 2014. 